See discussions, stats, and author profiles for this publication at: https://www.researchgate.net/publication/286694550

\title{
Sclera recognition using dense-SIFT
}

Conference Paper · December 2013

DOI: 10.1109/ISDA.2013.6920711

\section{CITATIONS}

28

4 authors, including:

Abhijit Das

University of Southern California

42 PUBLICATIONS 301 CITATIONS

SEE PROFILE

Miguel A. Ferrer

Universidad de Las Palmas de Gran Canaria

281 PUBLICATIONS 2,978 CITATIONS

SEE PROFILE

Some of the authors of this publication are also working on these related projects:

HOCR in Bangla View project

Project Document image retrieval View project mapada Pal

Indian Statistical Institute

397 PUBLICATIONS 7,299 CITATIONS

SEE PROFILE 


\title{
Sclera Recognition Using Dense-SIFT
}

\author{
Abhijit Das $^{\mathrm{a}}$, Umapada Pal ${ }^{\mathrm{b}}$, Michael Blumenstein ${ }^{\mathrm{a}}$ and Miguel Angel Ferrer Ballester ${ }^{\mathrm{c}}$ \\ ${ }^{\mathrm{a}}$ Institute for Integrated and Intelligent Systems, Griffith University, Queensland, Australia \\ Email: abhijit.das@griffithuni.edu.au,m.blumenstein@griffith.edu.au \\ ${ }^{\mathrm{b}}$ Computer Vision and Pattern Recognition Unit, Indian Statistical Institute, Kolkata, India, Email: umapada@isical.ac.in \\ ${ }^{\mathrm{c}}$ IDeTIC, University of Las Palmas de Gran Canaria, Las Palmas, Spain, Email: mferrer@ dsc.ulpgc.es
}

\begin{abstract}
In this paper we propose a biometric sclera recognition and validation system. Here the sclera segmentation is performed by time adaptive active contour-based region growing technique. The sclera vessels are not prominent so image enhancement is required and hence a bank of $2 \mathrm{D}$ decomposition Haar wavelet multi-resolution filters is used to enhance the vessels pattern for better accuracy. For feature extraction Dense Scale Invariant Feature Transform (D-SIFT) is used. D-SIFT patches descriptors of each training images are used to form bag of feature by using k-means clustering and spatial pyramid model, which is used to produce the training model. Support Vector Machine (SVM) is used for classification. UBIRIS version 1 dataset is used here for experimentation. An appreciable amount Equal Error Rate (EER) of $0.66 \%$ is achieved in the experiment.
\end{abstract}

Keywords-Biometric; Sclera vessels Patterns; D-SIFT; SVM; Bag of feature, $k$ - means, Bank of $2 D$ decomposition Haar multiresolution filters wavelet.

\section{INTRODUCTION}

Security of identification an individual is one of the greatest threats for today's world. As Biometrics is the science of identifying or verifying every individual in a set of people uniquely by using physiological or behavioral characteristics possessed by the user, so biometric will be s a good key to solve such problem. Opposed to the knowledge-based and token-based security systems, cutting-edge biometrics-based identification systems offer higher security and less probability of spoofing. The need of biometric systems is increasing in day-to-day activities due to its ease of use by common people, e.g. in attendance system of organizations, citizenship proof, door lock for high security zones etc. The financial sectors, government, reservation systems are adopting biometric technologies for ensuring security in their own domains and to maintain signed activity log of every individual. Biometric earliest cataloging of fingerprints dates back to 1891 when Juan Vucetich started a collection of fingerprints of criminals in Argentina. First automatic biometric system was proposed in 1980's. Till date various biometric system has been proposed. Though biometric identifiers such as digital fingerprints, retinal scans, facial characteristics, gait, and vocal patterns are distinctive to each and every person and are considered more reliable and capable than the traditional token-based or knowledge-based technologies in differentiating between an authorized and a fraudulent person, still no biometric system is a perfect biometric system.
Among various biometrics, the ocular biometrics including iris and retina are known as among the most accurate biometrics. But few disadvantages like capture of iris image requires cooperation of user since off-axis iris image can deteriorate the system performance, and retina scanning requires contact with eye-piece which is far from being userfriendly. Apart from iris and retina, the human eye has an ocular surface known as the sclera. Till date, this biometric is relatively less studied and little is known regarding its usefulness.

The first recognized work on sclera biometrics is recorded in [1]. In this paper, the authors discuss methods for conjunctival image pre-processing by computing a Gaussian filters and Hessian matrix. In order to derive a suitable vascular template for biometric authentication, feature extraction is performed by a minutiae template.

In [2], the authors have explained the possible use of texture portions of the sclera as a biometric. The Discrete Cosine Transform and wavelets are used for feature representation. A feed forward Neural Network with a single hidden layer is used here for classification.

In [3], a multi-angled sclera recognition technique is proposed. A Gabor filter is used here for sclera vessel enhancement. A polar coordinate-based mapping with iris center was performed for template matching.

In [4] a multimodal eye recognition technique is proposed using sclera and iris. Here Gabor enhancement and line description are used for feature extraction. And for classification, the Hamming distance is used for templatebased matching.

A new method of sclera segmentation for both grey and color images is proposed in [5]. Otsu's binarization method is used for segmenting the grey-level images, and for color images, the HSV model is used for segmentation.

A quality fusion-based biometric technique using sclera and iris information is explained in [6]. Here a quality function is defined for both iris and sclera in order to combine them.

A new method of sclera segmentation by a time adaptive active contour-based method is explained in [7]. A sclera recognition technique for multi-spectral occluded sclera recognition is proposed in [8]. Coarse-based sclera segmentation is performed here to get the region of interest. Then Speed up Robust Features (SURF) are used for feature representation. Key point matching, pixel to pixel and blood vessel bifurcation matching is used for classification of sclera matching. 
In [9], GLCM (Gray Level Co-occurrence Matrix) is used for sclera biometrics. Statistical features of GLCM, such as contrast, correlation, energy and homogeneity, were used in conjunction with Fisher linear discriminant analysis and regularized neural network classifiers in order to recognize textures arising from conjunctival vessels. Match score level fusion of Fisher LDA and neural networks are used to provide the best results.

LBP (Local Binary Pattern) feature is used for sclera biometrics in [10]. Here a histogram equalization-based HSV image of the sclera is used for segmentation. A mask is developed to search the overlapping region for a template match.

A multimodal eye recognition technique is explained in [11]. A weighted fusion scheme to combine the information originating from these two modalities is highlighted here.

A feasibility study on sclera recognition is performed in [12]. This feasibility study is undertaken with respect to global and local information, which are extracted from the periocular region using texture and point operators resulting in a feature set that can be used for matching.

In [13], the authors propose a comprehensive sclera image quality measure which can quickly detect if the image has a valid eye, assess the image quality, evaluate the segmentation accuracy, and measure if the image has sufficient feature information for recognition.

In [14], the authors have designed enhancement and registration methods to process and match conjunctival vasculature obtained under non-ideal conditions. The goal is to determine if conjunctival vasculature is a viable biometric in an operational environment.

This work proposes a whole biometric system for personal identification based on sclera vessels. Here the sclera segmentation is performed by Fuzzy C-means clustering. We propose a new preprocessing for vein highlighting by Discrete Meyer wavelet. The sclera feature extraction based on Dense Scale Invariant Feature Transform (D-SIFT) is also new at the literature. Support Vector Machine (SVM) is used for classification.

The organization of the paper is as follows: - Section II explains the proposed approach of segmentation, preprocessing of the sclera images, followed by the sclera vessel enhancement process, feature extraction and classification. In Section III the experimental details are given and Section IV draws the overall conclusions.

\section{PROPOSED APPROCH}

In this section, at first, the proposed sclera segmentation process, a sclera vein enhancement technique, and feature extraction of sclera texture patterns are explained. Next, classification technique is discussed.

\section{A. Sclera Segmentation}

The sclera is a white region of connective tissue and blood vessels surround the iris. This portion of blood vessels inside the sclera region is randomly-oriented which creates a pattern. This pattern is used for biometric identification. Segmentation is the first step for most biometric related research. Similarly in sclera biometric a perfect segmentation is important otherwise, an incorrect segmentation can reduce the pattern available, but also it can introduce other patterns such as eyelashes and eyelids. Generally the portion of blood vessels inside the sclera region is randomly-oriented which creates a pattern. As they are oriented in different layers, the intensity of the vessels varies highly. Some of them are quite bright and bundled. And this creates a hindrance to standard region growing. So we have considered the red channel of the image for segmentation, as the blood vessels are less prominent here as shown in figure 1(b). A representation for each color channel is indicated in Figure 1.

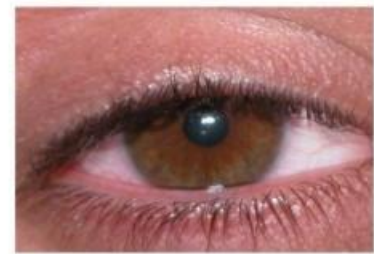

$1(a)$

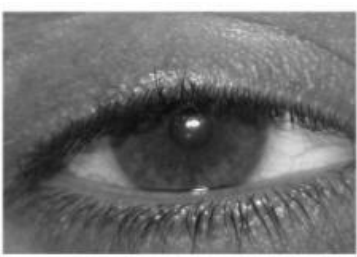

$1(\mathrm{c})$

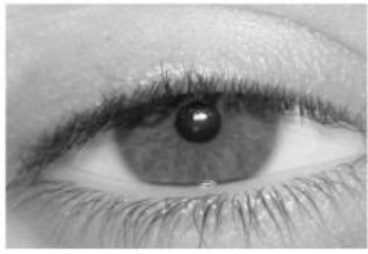

1(b)

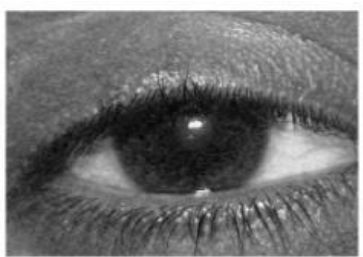

$1(\mathrm{~d})$
Figure. 1. (a) The image of the original RGB image, (b) The red channel component of 1(a), (c) The Green channel component of 1(a), and (d) blue channel component of 1(a),

Adaptive histogram equalization is performed with a small window of $2 \times 2$ to reduce the vessel content. Next to which we use a bank of low pass Haar reconstruction filters to get a clear white sclera without the vessel. The filter is used with a high cut off. Analyzing different results, the cut off value that produces the best result is determined and used for experimentation. The figure 2(a) is the histogram equalized image and 2(b) the Haar filtered image of 2(a).

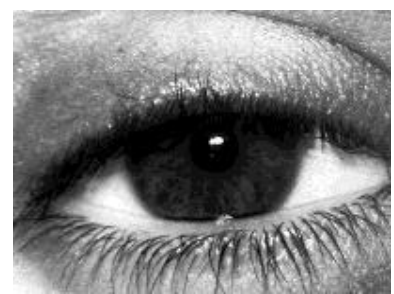

2(a)

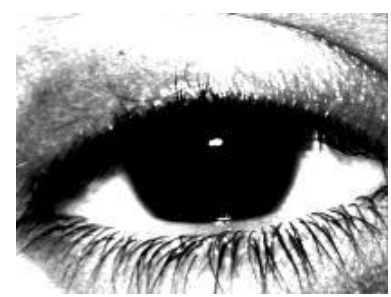

2(b)
Figure. 2. (a) is the histogram equalized image and (b) the Haar filtered image of 2(a). 
This preprocessed image can be used for segmentation by a time adaptive active contour-based region growing segmentation method discussed in [15]. The right and the left sclera are segmented separately. For region growing-based segmentation, we need a seed point. In order to get the seed point we use Daugmans integro-differential method [16], which is used to calculate the center of the iris. From the center of the iris at a distance of 1.1 of the radius length of the iris and a deviation of 45 degrees with the horizontal, the seed point for region growing is set in both sides of the sclera as explained in Figure 3.

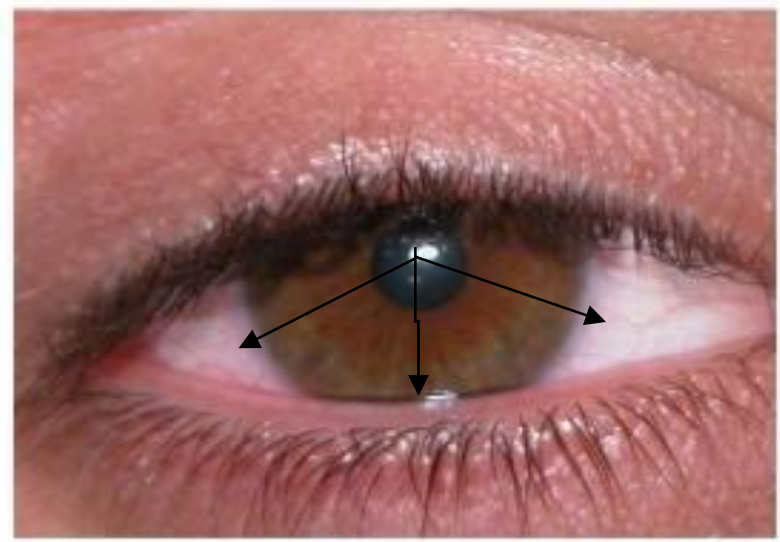

Figure. 3. Seed point for sclera segmentation

Now the seed point grows to provide the total sclera region as explained in Figure 4.

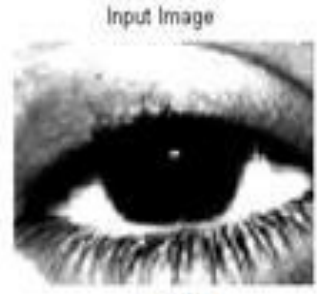

4(a)

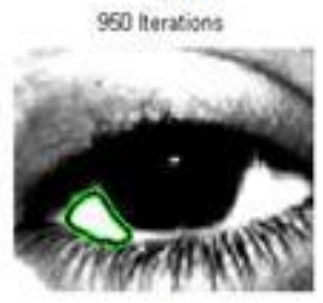

$4(c)$

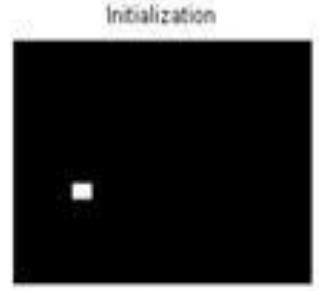

$4(b)$

Global Region-Based Segmentation

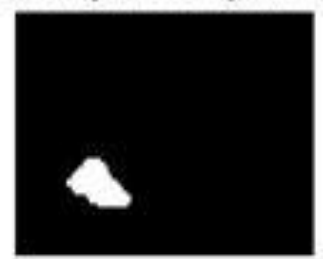

$4(d)$

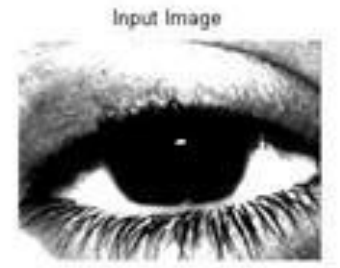

$4(e)$

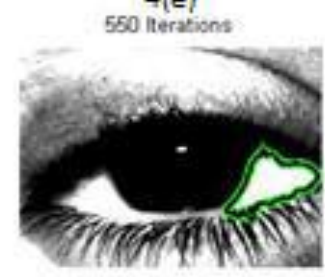

$4(\mathrm{~g})$

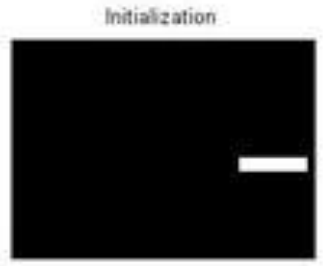

4(f)

Global Region Bused Segmentation

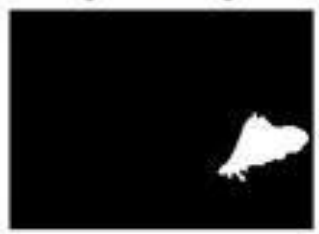

$4(h)$
Figure. 4. Region growing segmentation method of left and right sclera. 4(a) The Histogram equalized and filtered image of red component of 1(a),4(b) Initial size of the seed for right sclera,4(c) Segmented image of 4(a),4(d) Segmented mask developed for right sclera, 4(e)The Histogram equalized and filtered image of red component of 1(a),4(f) Initial size of the seed for left sclera,4(g) Segmented image of 4(e), 4(h) Segmented mask developed for left sclera

\section{B. Sclera vessel structure enhancement}

The vessels in the sclera are not prominent, so in order to make them clearly visible, image enhancement is required. Adaptive histogram equalization is performed with a window size of $42 \times 42$ on the green channel of the sclera image (as the sclera vessels patter are most prominent in the green channel), to make the vessel structure more prominent as shown in Figure 5.

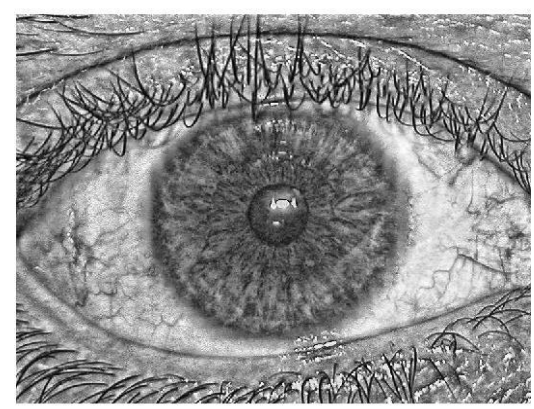

Figure 5: Adaptive histogram equalization of sclera image.

A bank of 2D decomposition Haar wavelet multi-resolution filters is used to enhance the vessels pattern. Figure 6 shows the vessel enhanced image. 


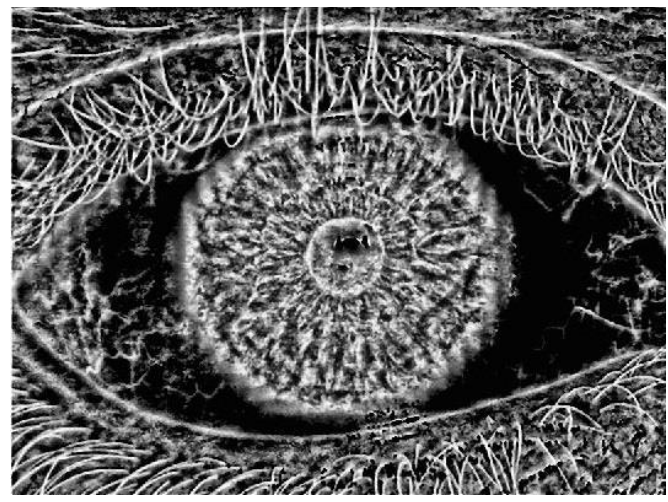

Figure 6: The vessel enhanced image.

\section{Feature Extraction Method}

In last years, the local descriptors emerge as a way to improve feature extraction methods. It also worked efficiently in presence of distortion such as of scale, rotation, translation and occlusion. Its high discriminative capability and robustness attracted researchers in the area of biometrics. The variance of position of eyelids produces occlusions which are difficult to manage with traditional texture feature methods. The robustness against occlusion is one of the most interesting factors in the application on sclera recognition. The local descriptor method applied in this paper is the Scale Invariant Feature Transform (SIFT). The SIFT was originally proposed in [17] and it was used for biometrics such as palm print [18], iris [19], among others. The sclera feature extraction based on Dense Scale Invariant Feature Transform (D-SIFT) was performed here. D-SIFT patches descriptors of each training images are used to form bag of words, which is used to produce the training model. Here, 128-dimensional based SIFT key point was extracted which stores the gradients of $4 \times 4$ locations around a pixel in a histogram bin of 8 directions. The gradients of each key point are aligned to the main direction, which makes it a rotation invariant descriptor. Different Gaussian scale spaces are considered for the computation of vector to which makes it scale invariant. The blue cross in Fig. 7 represent the $16 \times 16$ SIFT patches and Fig. 8 shows graphical representation of SIFT descriptors of 4 bins and 8 orientations. Next, k-means clustering technique is applied on the patches from the training set for the generation of codebook. The typical vocabulary size for our experiments is 1024. Descriptors from each training images are used with the code book to form bag of feature, which is used to produce the training model. The spatial pyramid matching is an extended version of bag-of-features (BoF) model; it is simple and computationally efficient. BoF model the spatial order of local descriptors is not considered, so it restricts the descriptive power of the image representation. Limitation of $\mathrm{BoF}$ is overridden in SPM [20] approach, which is successfully applied on image recognition tasks. An image is partitioned into $2^{i} \times 2^{i}$ segments where $i=0 ; 1 ; 2$, represents different resolutions.

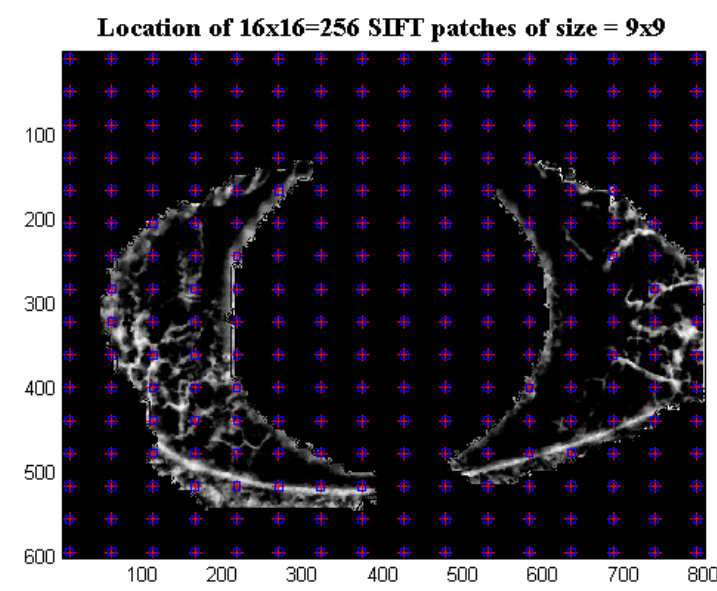

Figure 7: Image is divided into $16 \times 16$ location of $9 \times 9$ patch size for dense SIFT descriptor.

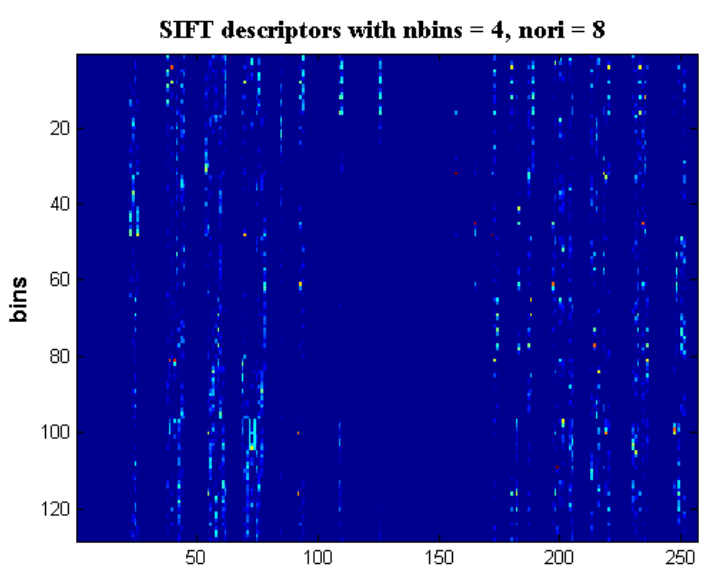

Figure 8: SIFT descriptor with number of bins and orientation

Next, the BoF histograms are computed within each of the $2^{i}$ segments, and all the histograms are finally merged to form a vector representation of the image. SPM reduces to BoF, when the value of the scale $i=0$. Here, the pyramid matching is performed in two-dimensional image space and use a traditional clustering technique in feature space. The number of matches at level $i$ was given by the histogram intersection function:

$$
I(g x ; g Y)=\sum_{k=i}^{n} \min \left(g x(i) ; g_{Y}(i)\right)
$$

Finally, the representation of the image for classification is the total number of matches from all the histograms, which is given by the definition of a pyramid match kernel:

$$
K(X ; Y)=\sum_{i=1}^{l} 0.5^{i}\left(l_{i}-l_{i-1}\right)
$$

All total $21(16+4+1)$ BoF histograms are computed from these three levels, and all the histograms are concatenated to get the final vector representation of an image. The equation below represents the pyramid match kernel for three scales: 


$$
K \Delta=I_{2}+0.5\left(I_{1}-I_{2}\right)+0.25\left(I_{0}-I_{1}\right)
$$

\section{Classification}

Support Vector Machine (SVM) is used for classification. A SVM is a popular supervised machine learning technique which performs an implicit mapping into a higher dimensional feature space. This is also known as kernel trick. After the mapping is completed it finds a linear separating hyper plane with maximal margin to separate data from this higher dimensional space. Library for Support Vector Machines (LIBSVM) is used for SVM implementation. Though new kernels are being proposed, the most frequently used kernel functions are linear, polynomial, and Radial Basis Function (RBF). This study uses the RBF kernel.

SVM or LIB-SVM makes binary decision and multi-class classification for personal identification has been made in this study by adopting the one-against all techniques. We carried out grid-search on the hyper-parameters in the 5 -fold cross validation for selecting the parameters on the training sequence. The parameters setting that produce the best crossvalidation accuracy were picked.

\section{EXPERIMENTAL RESULT}

The experimental setup and the results of our proposed work are explained in this section.

\section{A. Data Set}

In order to evaluate the performance of our proposed method, the UBIRIS v1 database [21] is utilized for our experiments. This database consists of 1877 RGB images taken in two distinct sessions (1205 images in session 1 and 672 images in session 2) from 241 identities where each channel of RGB color space is represented in grey-scale. The database contains blurred images and images with blinking eyes. Both high resolution images $(800 \times 600)$ and low resolution images $(200 \times 150)$ are provided in the database. All the images are in JPEG format. We have used different quality images and some of the sample images are shown below in Figure 9. Some of them are not occluded having good quality of sclera regions visible, some of them are of medium quality and the third type is of poor quality with respect to sclera region visibility. Some of the sample images used in the experiment is given in Figure 9.

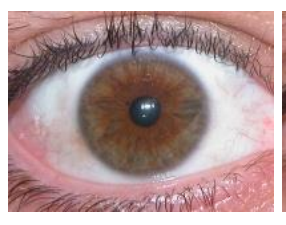

9(a)

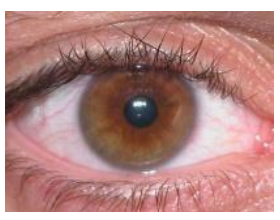

9(b)

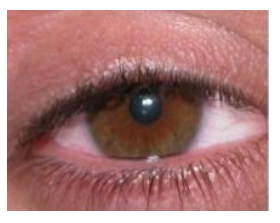

$9(\mathrm{c})$
Figure 9: Different quality of eye image used. 9(a) is the type of best quality image, 9(b) ) is the type of medium quality \& 9(c) is the type of Poor quality
For our experimentation we have considered the images of session 1. Three images from the session is randomly chosen and utilized for training model generation and the other two were used as query images. So we have $241 * 2$ scores for FRR and $242 * 241$ score for FAR statistics. In our experiment we have also used some closed images examples of the closed images are given below in figure 10 .
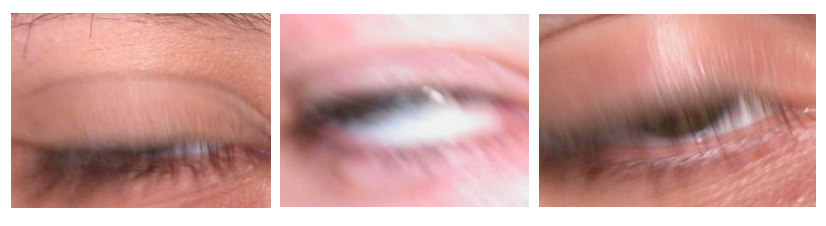

Figure 10: Example of closed eyes

All the stimulation experiments performed here were developed in Matlab.

\section{B. Results}

The experiments have been conducted to work out the sclera identification capability of the proposed features and classifier. As it is a classical identification or verification problem, the results will be given in terms of receiver operating characteristic (ROC) curve. It has been found that the proposed technique produces high recognition accuracy of $99.34 \%$. The result can be reflected from the ROC curve in the Figure 11. Along the $\mathrm{Y}$ - axis is the GAR (Genuine Acceptance Rate) and along the X-axis the False Acceptance Rate (FAR). In the experiment an Equal Error Rate of $0.66 \%$ is achieved, this indicates the verification significance of the proposed technique.

It was been found that the proposed system is also capable of working on the poor quality of images where partial part of the sclera is visible. So this also indicates that the system is robust with respect to the environmental conditions.

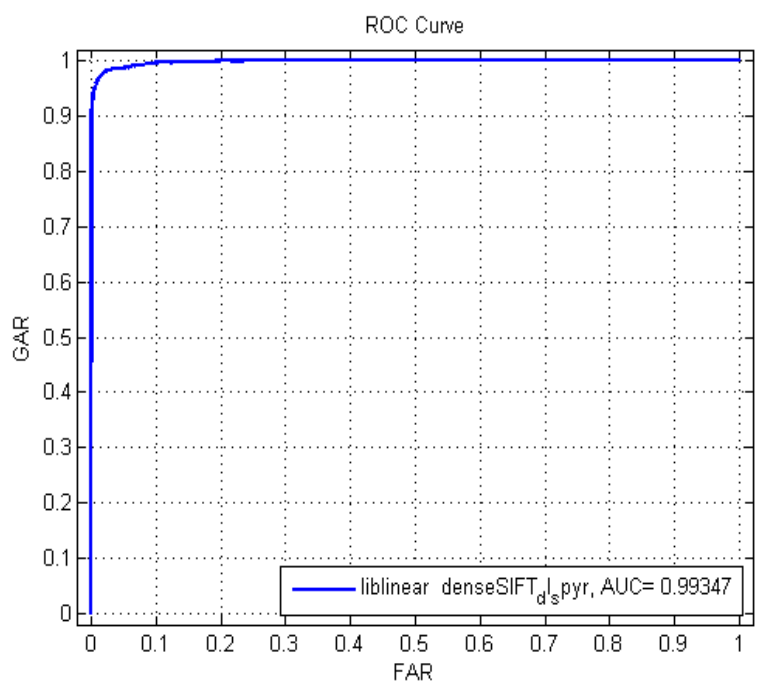

Figure 11: ROC curve of the recognition experiment 


\section{Comparison of results}

The results of the proposed work is put in perspective with the state-of-the-art by analyzing it with the most similar work on UBIRIS version 1 , we could find in the literature. Table 1 reflects a state-of-the-art comparative analysis of the most similar work on UBIRIS version 1 . From the table it can be seem that the method due to $\mathrm{Oh}$ and Toh et al. [10] gives a slight better result than our proposed method. They used manual sclera segmentation for some images and that is why they obtained better results than ours. On the other hand, the proposed method gives better results than Zhou et al. [5].

Table 1. The state-of-the-art comparative results on UBIRIS version 1

\begin{tabular}{|c|c|}
\hline Work & Equal Error Rate (in \%) \\
\hline Zhou et al. [5] & 1.34 \\
\hline Oh and Toh et al. [10] & $\begin{array}{c}0.47 \text { (manual segmentation for } \\
\text { some images) }\end{array}$ \\
\hline Proposed System & 0.66 \\
\hline
\end{tabular}

In [5] very poor quality (e.g., blur, blink, or no sclera-area image) cannot be segmented by segmentation method. But our proposed technique is able to segment most of the poor quality, except the totally closed eye images (examples of such images are shown in figure 10). In [10] manual points marking and connecting were performed on 333 images with unsuccessful sclera region localization. Hence our segmentation technique outperformed the other previous segmentation technique. In the previous approaches classification was done by template matching, to best of our knowledge this is the first paper in the literature on sclera biometrics where statistical classifier, that is SVM is used which is more reliable than the other classifier used previously in literature.

\section{CONCLUSIONS}

This paper has proposed a novel method of sclera recognition. We have proposed a sclera preprocessing algorithm to whiten the sclera eye portion, which improves the accuracy of the active contour procedure proposed for sclera segmentation. The vessel pattern has been enhanced with adaptive histogram equalization and a high pass Haar filter for establishing appropriate features. The D-SIFT based descriptor is used for feature extraction. K-means clustering technique is used for generating the code book. The proposed approach has achieved high recognition accuracy employing the UBRIS version1 dataset.

\section{References}

[1] R. Derakhshani, A. Ross, and S. Crihalmeanu. A new biometric modality based on conjunctival vasculature. Proceedings of Artificial Neural Networks in Engineering: 1-8, 2006.

[2] R. Derakhshani and A. Ross. A texture-based neural network classifier for biometric identification using ocular surface vasculature. Proceedings of International Joint Conference on Neural Networks: 2982-2987, 2007.

[3] Z. Zhou, Y. Du, N. L. Thomas, and E. J. Delp. Multi angled sclera recognition. IEEE Workshop on Computational Intelligence in Biometrics and Identity Management: $103-108,2011$.

[4] Z. Zhou, Y. Du, N. L. Thomas, and E. J. Delp. Multimodal eye recognition. Proceedings of the International Society for Optical Engineering, 7708(770806):1-10, 2010.

[5] Z. Zhou, Y. Du, N. L. Thomas, and E. J. Delp. A new biometric sclera recognition. IEEE transaction on System, Man And Cybernatics -PART A: System And Human, 42(3): 571-583,2012.

[6] Z. Zhou, Y. Du, N. L. Thomas, and E. J. Delp, Quality Fusion Based Multimodal Eye Recognition, IEEE International Conference on Systems, Man, and Cybernetics : 1297-1302, 2012.

[7] M. H. Khosravi and R. Safabakhsh, Human eye sclera detection and tracking using a modified time-adaptive self-organizing map, Pattern Recognition, 41 : 2571 - 2593, 2008.

[8] S. Crihalmeanu and A. Ross, Multispectral sclera patterns for ocular biometric recognition, Pattern Recognition Letters, 33: 1860-1869, 2012.

[9] S. Pavan Tankasala, P. Doynov, R. R. Derakhshani, A. Ross and S Crihalmeanu, Biometric Recognition of Conjunctival Vasculature using GLCM Features, International Conference on Image Information Processing : 1-6, 2011.

[10] K. Oh and K. Toh, Extracting Sclera Features for Cancelable Identity Verification, 5th IAPR International Conference on Biometric: 245-250, 2012.

[11] V. Gottemukkula, S. K. Saripalle, S. P. Tankasala, R. Derakhshani, R. Pasula and A. Ross, Fusing Iris and Conjunctival Vasculature: Ocular Biometrics in the Visible Spectrum, IEEE Conference on Technologies for Homeland Security: 150-155, 2012.

[12] U. Park, A. Ross, and A. K. Jain, Periocular Biometrics in the Visible Spectrum: A Feasibility Study, IEEE 3rd International Conference on Biometrics: Theory, Applications, and Systems: 1-6, 2009.

[13] Z. Zhou, E. Y. Du, and N. L. Thomas, A Comprehensive Sclera Image Quality Measure, 11th International Conference on Control, Automation, Robotics and Vision: 638-643, 2010.

[14] S. Crihalmeanu , A. Ross , and R. Derakhshani, Enhancement and Registration Schemes for Matching Conjunctival Vasculature, Appeared in Procceding of the 3rd IAPR/IEEE International Conference on Biometrics :1247-1256, 2009

[15] T. F. Chan, and L. A. Vese, Active Contours Without Edges, IEEE Transaction On Image Proccessing 10( 2): 266-277, 2001

[16] J. G. Daugman. High confidence visual recognition of persons by a test of statistical independence. IEEE Transactions on Pattern Analysis and Machine Intelligence, 15(11):1148-1161, 1993.

[17] D. G. Lowe, 'Distinctive image features from scale-invariant keypoints', Proceedings of IJCV 2004, 2 (60), 91-110, 2004.

[18] M. Morales, M. A. Ferrer and A. Kumar. "Toward Contactless Palmprin Authentication". IET Computer Vision 2011, 5(6), pp. 407-416.

[19] F. Alonso-Fernandez, P. Tome-Gonzalez, V. Ruiz-Albacete, J. OrtegaGarcia: Iris recognition based on sift features. In: Int'l Conf. on Biometrics, Ident. and Sec (BIdS), 1-8, 2009.

[20] S. Lazebnik, C. Schmid, and J. Ponce: Beyond bags of features: Spatial pyramid matching for recognizing natural scene categories, In Proc. Computer Vision and Pattern Recognition (CVPR), 2, 2169-2178.

[21] UBIRIS version 1 dataset for eye, Available at, (http://iris.di.ubi.pt) 